\title{
Essai de valorisation d'Azolla (Azolla microphylla Kaulf) dans la production porcine en zone humide
}

\author{
J-M. M. ACCODJI, E. D. FIOGBE * et K. H. GANGBAZO \\ Unité de Recherche sur les Zones Humides (URZH), Département de Zoologie et Génétique, Université \\ d'Abomey-Calavi, 01 BP 526 Cotonou, Bénin. \\ *Auteur correspondant, Tél./Fax:229.21361076, E-mail: edfiogbe@yahoo.fr
}

\section{RESUME}

Une étude sur l'effet de la substitution partielle d'une provende commercialisée pour porc en croissance par Azolla frais a été menée dans une porcherie moderne. Au total 16 porcelets de race locale (8 mâles et 8 femelles) mis en lots de 4 ont été soumis à 4 formules alimentaires dont 3 expérimentales et 1 témoin. La formule 1 est donc une provende standard de performances zootechniques connues de tous les éleveurs de porcs (témoin). Les formules 2, 3 et 4 ne sont rien d'autre que la formule témoin substituée respectivement par 15\%; $30 \%$ et $45 \%$ Azolla. Des différences significatives ont été observées notamment entre la formule 1 (témoin) et la formule $2(15 \%$ Azolla $)$. Cette dernière a présenté un gain pondéral et une efficacité de conversion alimentaire significativement $(\mathrm{P}<0.05)$ plus élevés que la formule témoin. Les résultats obtenus dans cette étude semblent indiquer que la provende entière pour porc en croissance substituée par Azolla frais à $15 \%$ d'incorporation peut améliorer significativement les performances zootechniques des porcelets nourris, et permettre par cette même occasion une rentabilité économique certaine, Azolla étant produit de façon marginale dans des étangs en communication avec le lisier des porcs.

(c) 2009 International Formulae Group. All rights reserved.

Mots clé : Azolla frais, croissance, porcelets, provende, ration, substitution.

\section{INTRODUCTION}

Au Bénin, l'élevage porcin bien que réalisé à grande échelle en milieu urbain et périurbain, reste cependant mal connu en zone rurale et surtout humide à cause du coût et de la disponibilité des matières premières. L'effectif porcin béninois est estimé à plus de 581600 têtes en 1996 avec une augmentation de $3 \%$ du cheptel les quatre dernières années (Fiogbé et Gangbazo, 2005). L'approche de solution la plus probante pour promouvoir son développement en zones rurales reste à coup sûr la mise en valeur des matières premières locales non encore valorisées. En effet, le porc est un excellent assimilateur, à cause essentiellement de son tube digestif composé d'un intestin qui fait quatorze fois la longueur de son corps, soit 20 mètres chez un porc de 100 kilogrammes (Serres, 1989). L'essentiel $\mathrm{du}$ processus d'absorption se produit dans l'intestin grêle où les sucs digestifs secrétés par le pancréas, le foie et l'intestin grêle parachèvent le processus de digestion déclenché depuis la bouche de l'animal (Holnes, 1994). C'est dans cet ordre d'idées que, pour promouvoir l'élevage porcin en zones rurales et surtout en zones humides, nous nous sommes permis d'introduire Azolla, cette fougère aquatique naturellement riche en protéines (Van Hove, 1989), non coûteux et largement disponible en zones humides, dans l'alimentation des porcs en vu de mieux rentabiliser leur élevage. Le mode de multiplication de la fougère Azolla reste 
dominé par la multiplication végétative. Cependant, la reproduction sexuée est possible et se manifeste par la germination de spores dont la formation est sous la dépendance de plusieurs facteurs. Herd et al. (1989) ont observé que la sporulation chez Azolla est régulée par les effets des interactions entre la photopériode, l'intensité lumineuse, la température, le $\mathrm{pH}$ et la quantité d'azote et de phosphore.

\section{MATERIEL ET METHODES \\ Les animaux}

Le matériel animal destiné à

l'expérimentation est constitué de 16 porcelets de race locale dont huit (8) mâles et huit (8) femelles âgés de 64 jours. Le poids moyen de chaque lot était $17,7 \pm 0,2 \mathrm{~kg}$. Tous les animaux ont été marqués individuellement de façon qu'on puisse les identifier.

\section{Site expérimental}

L'expérience a été conduite sur le site expérimental agro - piscicole de l'Unité de Recherche sur les Zones Humides (URZH) du Département de Zoologie et Génétique de la Faculté des Sciences et Techniques (FAST) de l'Université d'Abomey-Calavi (U.A.C). Ce site se trouve dans la commune d'Akassato (Commune d'Abomey-Calavi), banlieue Nord de Cotonou $\left(6,12^{\circ}\right.$ latitude Nord, $2^{\circ} 26^{\prime}$ longitude Est) et situé au cœur des zones humides qui sont en bordure du lac Nokoué. Il y règne un climat de type sub - équatorial qui se caractérise par l'alternance de deux (2) saisons sèches et de deux (2) saisons pluvieuses. Cependant, l'existence d'étangs confère au site, un micro climat spécifique.

\section{Espèce d'Azolla utilisée}

L'espèce d'Azolla est Azolla microphylla. Sa valeur bromatologique est présentée sur le tableau 1.

\section{Aliments expérimentaux}

Ces différents régimes ont été formulés pour être iso caloriques conformément au niveau énergétique de la provende de base (formule 1). Un complément en huile rouge est donc apporté aux formules 2, 3 et 4 afin d'atteindre le niveau énergétique adéquat qui est de 2692,456 kcal/kg d'aliment (Tableau 2).

\section{Analyses statistiques}

Les paramètres d'utilisation des aliments et de croissance des porcs traités ont été comparés à la fin de l'expérience par l'analyse de variance à un critère de classification (ANOVA 1). Les paramètres tels que:

- $\quad$ le gain moyen quotidien GMQ

- l'indice de consommation IC

ont été calculés de la façon suivante :

$$
\operatorname{GMQ}(g / j)=\frac{P f-P i}{D t}
$$

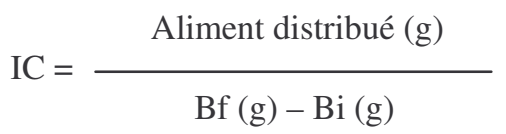

Où GMQ est le gain moyen quotidien, Pi et Pf sont les poids moyens initial et final des porcs par traitement, Bi et $\mathrm{Bf}$ les biomasses initiale et finale par traitement et dt la durée d'alimentation en jours.

Tableau 1: Valeurs bromatologiques de Azolla.

\begin{tabular}{lcc}
\hline Matière sèche (\%PF) & $4,9^{*}$ & $13,1^{* *}$ \\
Protéines brutes (N x 0,25) (\%) & $9,6^{*}$ & $36,6^{* *}$ \\
Matières grasses (\%PS) & $0,8^{*}$ & $6,7^{* *}$ \\
Fibres brutes (\%PS) & $2,5^{*}$ & $16,6^{* *}$ \\
Cendres (\%PS) & $9,0^{*}$ & $37,7 * *$ \\
Extrait sans azote (\%PS) & $31,1^{*}$ & $61,1^{* *}$ \\
Energie brute (kcal/kg) & $3270^{*}$ & $4240^{* *}$ \\
\hline \multicolumn{2}{c}{ PS : poids sec ; PF : poids frais ; $\mathrm{N}:$ azote ; $*$ valeur minimale ; ** = valeur maximale (Van Hove, 1989). }
\end{tabular}


Tableau 2: Composition centésimale des aliments expérimentaux.

\begin{tabular}{lllll}
\hline Matières premières & Formule 1 & Formule 2 & Formule 3 & Formule 4 \\
\hline Son de maïs & 46,20 & 39,46 & 32,49 & 25,53 \\
Son de blé & 11,61 & 9,86 & 8,12 & 6,38 \\
Tourteaux de palmiste & 29,01 & 24,66 & 20,30 & 15,95 \\
Huile rouge & 3,28 & 2,79 & 2,30 & 1,80 \\
Azolla & 0,00 & 15,00 & 30,00 & 45,00 \\
Farine de poisson & 4,83 & 4,11 & 3,38 & 2,65 \\
Coquille & 1,93 & 1,64 & 1,35 & 1,06 \\
Prémix & 1,93 & 1,64 & 1,35 & 1,06 \\
Sulfate de fer & 0,96 & 0,82 & 0,67 & 0,53 \\
Protéines brutes (\%) & 14,99 & 17,24 & 19,49 & 21,74 \\
Energie digestible & & & & \\
kcal/kg d'aliment & 2692,456 & 2558,58 & 2424,72 & 2290,85 \\
\hline
\end{tabular}

\section{RESULTATS}

\section{Performances zootechniques}

La quantité d'aliment ingérée par jour et par lot d'animaux a suivi une évolution croissante durant toute la période qu'a duré l'essai (Figure 1). Les consommations moyennes d'aliment par tête durant l'essai pour les lots 1, 2, 3 et 4 sont respectivement $0,36 \mathrm{~kg}, 0,38 \mathrm{~kg}, 0,31 \mathrm{~kg}$ et $0,27 \mathrm{~kg}$. Les quantités distribuées par lot durant tout l'essai sont de : $128,7 \mathrm{~kg}, 137,05 \mathrm{~kg}, 112,8 \mathrm{~kg}, 98,9$ $\mathrm{kg}$ respectivement pour les lots $1,2,3$ et 4 (Tableau 3).

Le poids moyen des porcelets par lot au début de l'expérimentation est de $4,45 \mathrm{~kg}$, $4,37 \mathrm{~kg}, 4,45 \mathrm{~kg}$ et $4,42 \mathrm{~kg}$ respectivement pour les lots $1,2,3$ et 4 . Ces poids initiaux ont connu une croissance lente durant toute la période d'expérimentation (Figure 2). Les valeurs moyennes à la fin de l'essai sont de $11,42 \mathrm{~kg}, 12,42 \mathrm{~kg}, 8,97 \mathrm{~kg}$ et $7,32 \mathrm{~kg}$ respectivement pour les lots $1,2,3$ et 4 .

L'analyse statistique de variance à un critère de classification ANOVA 1 des poids initiaux des porcelets en début d'expérimentation n'a révélé aucune différence significative entre les différents lots $(\mathrm{P}>0,05)$. Cependant, l'analyse statistique par ANOVA $1 \mathrm{du}$ poids des porcelets en fin d'expérimentation a montré une différence hautement significative entre les différents lots $(\mathrm{P}<0,01)$. Ainsi le lot 2 présente la meilleure croissance, suivi successivement des lots 1,3 et 4 .
La figure 3 traduit l'évolution du gain moyen quotidien par lot d'animaux selon la période d'expérimentation. Ce gain moyen quotidien (GMQ) est de 77,5 g/j, 89,44 g/j, $50,27 \mathrm{~g} / \mathrm{j}$ et $32,22 \mathrm{~g} / \mathrm{j}$ respectivement pour les lots $1,2,3$ et 4 .

Il est plus élevé dans le lot 2 que dans les autres lots. Le GMQ des quatre lots confondus est de 62,35 g/j par aliment.

L'analyse statistique par ANOVA 1 a par ailleurs montré une différence significative $(\mathrm{P}<0,05)$ entre les différents lots pour le GMQ.

La figure 4 représente les indices de consommation en fin d'expérimentation. Ces indices de consommation sont les mêmes $(4,22)$ pour les lots 1 et 2 , puis 6,30 et 9,86 respectivement pour les lots 3 et 4 . La valeur la plus élevée $(9,86)$ de l'indice de consommation (IC) est obtenue dans le lot 4 tandis que sa valeur la plus faible est obtenue dans les lots 1 et 2 .

\section{DISCUSSION}

\section{Niveaux énergétique et azoté des régimes alimentaires}

Les quatre formules alimentaires expérimentées sont iso caloriques. L'énergie digestible calculée pour les différents régimes alimentaires expérimentaux est de 2692,456 $\mathrm{kcal} / \mathrm{kg}$ d'aliment. Ce taux énergétique est inférieur de 13,2\% à celui de Houanye (1996). Il est également inférieur à ceux recommandés 
Tableau 3: Apports énergétiques complémentaires aux différentes formules.

\begin{tabular}{lllll}
\hline & Formule 1 & Formule 2 & Formule 3 & Formule 4 \\
\hline $\begin{array}{l}\text { Energie nécessaire } \\
\text { kcal/kg d'aliment }\end{array}$ & 2692,456 & 2692,456 & 2692,456 & 2692,456 \\
\hline $\begin{array}{l}\text { Energie disponible } \\
\text { kcal/kg d'aliment }\end{array}$ & 2692,456 & 2558,58 & 2424,72 & 2290,85 \\
\hline $\begin{array}{l}\text { Energie complémentaire } \\
\text { kcal/kg d'aliment }\end{array}$ & 0 & 133,86 & 267,73 & 401,60 \\
\hline $\begin{array}{l}\text { Valeur équivalente } \\
\text { En huile rouge (ml) }\end{array}$ & 0 & 15,75 & 31,5 & 47,25 \\
\hline
\end{tabular}

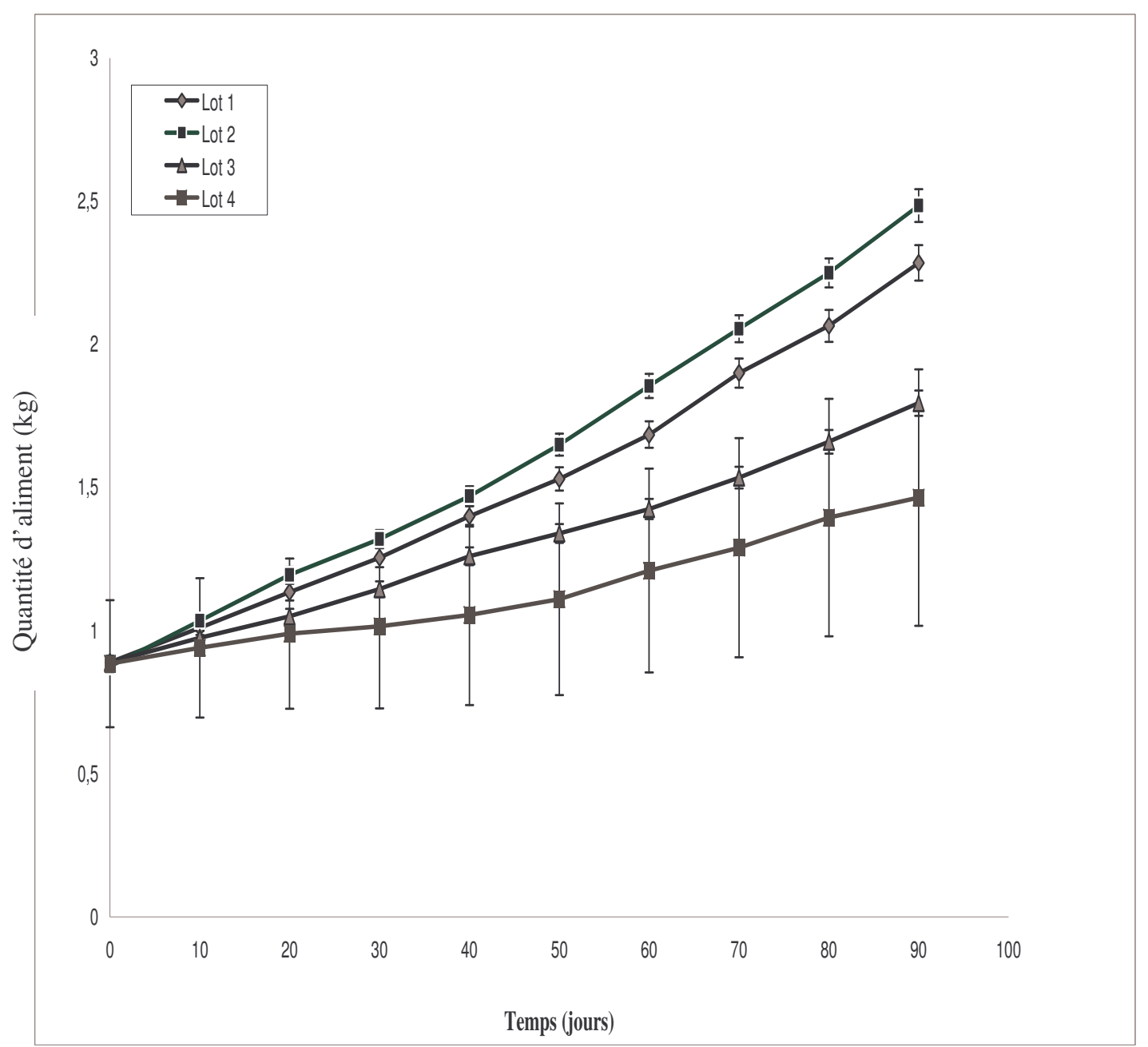

Figure 1: Evolution de la consommation journalière d'aliment sec par lot d'animaux. 


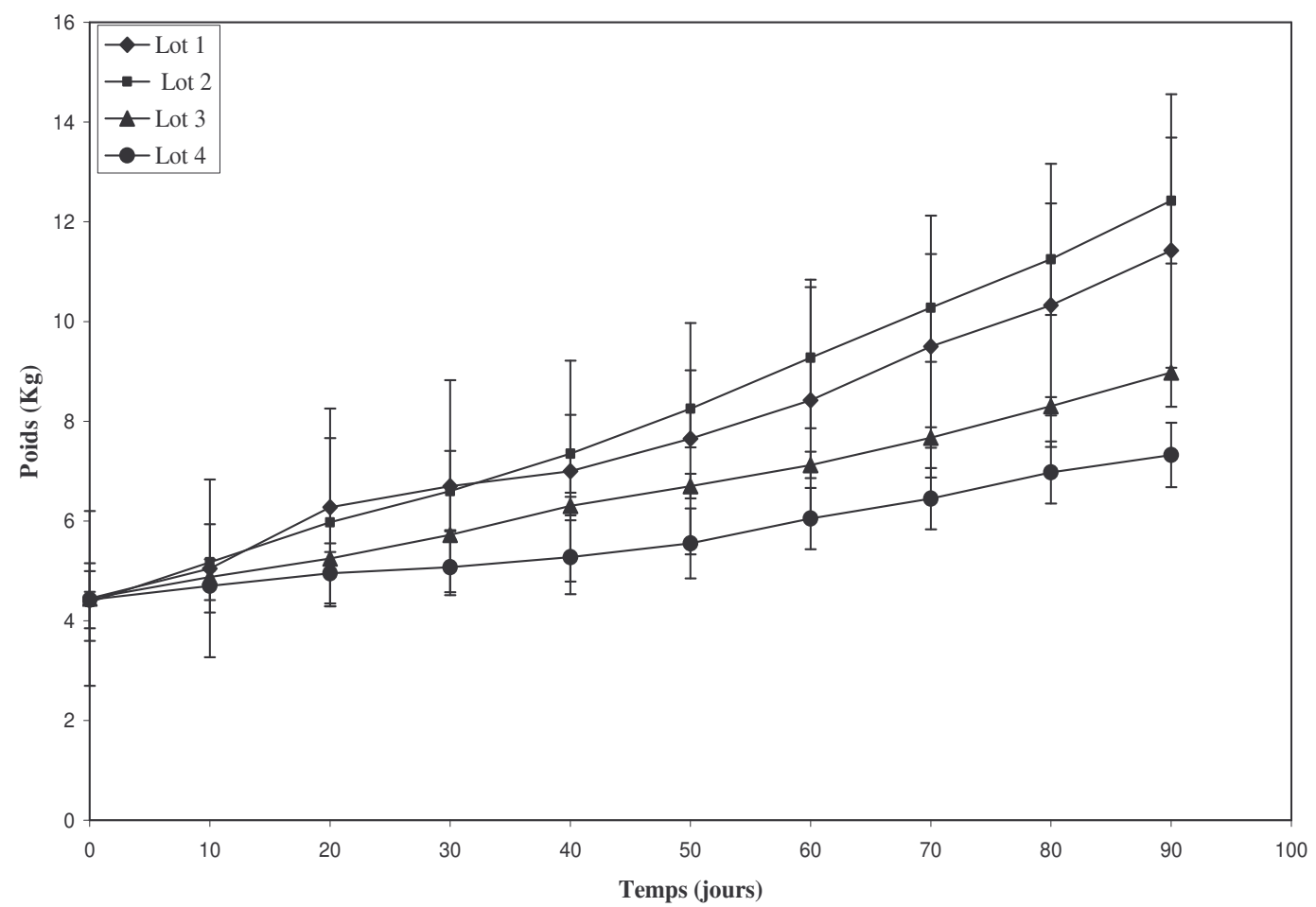

Figure 2: Evolution du poids moyen des porcs par lot.

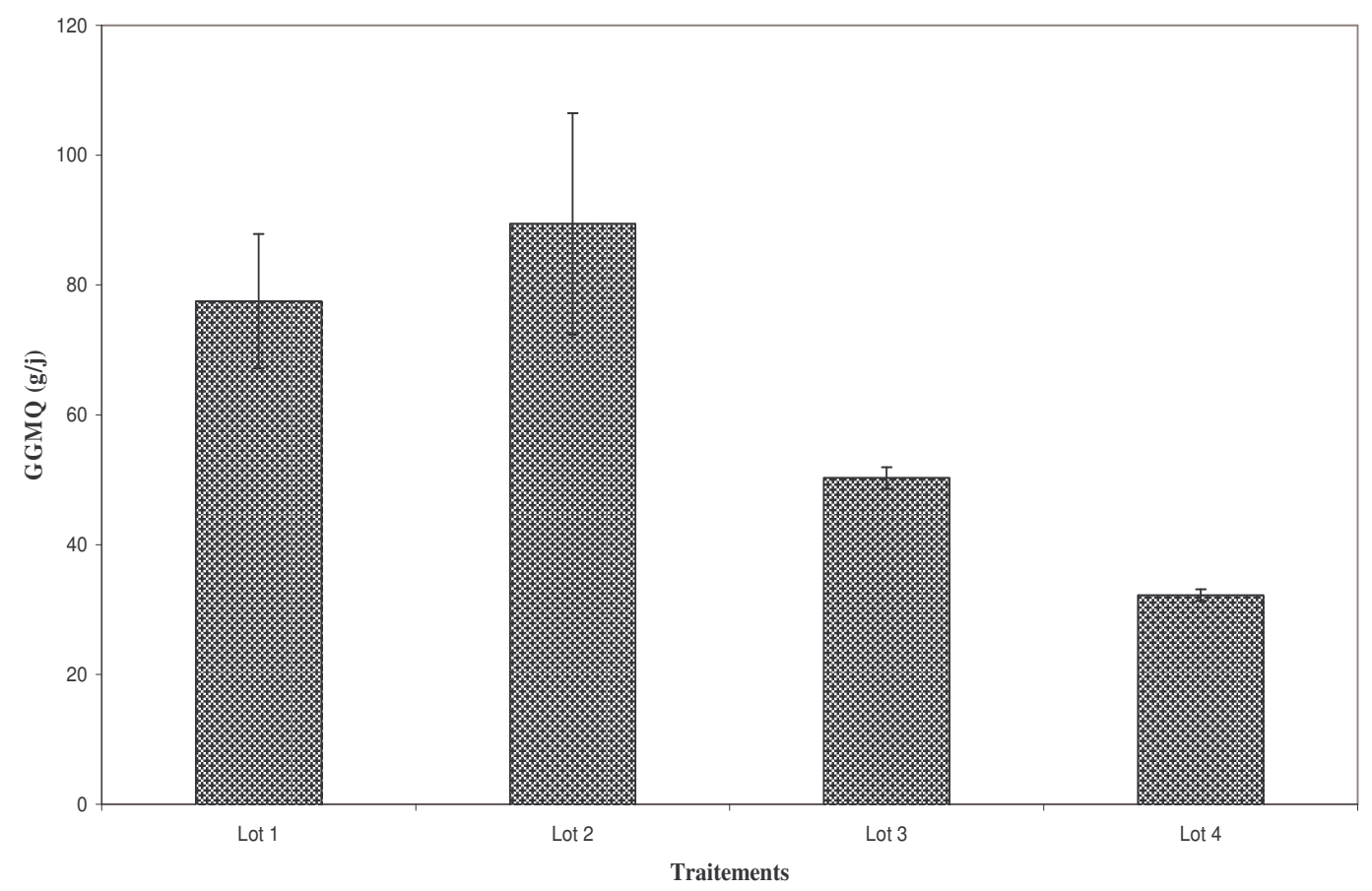

Figure 3: Histogrammes des gains moyens quotidiens des différents lots. 


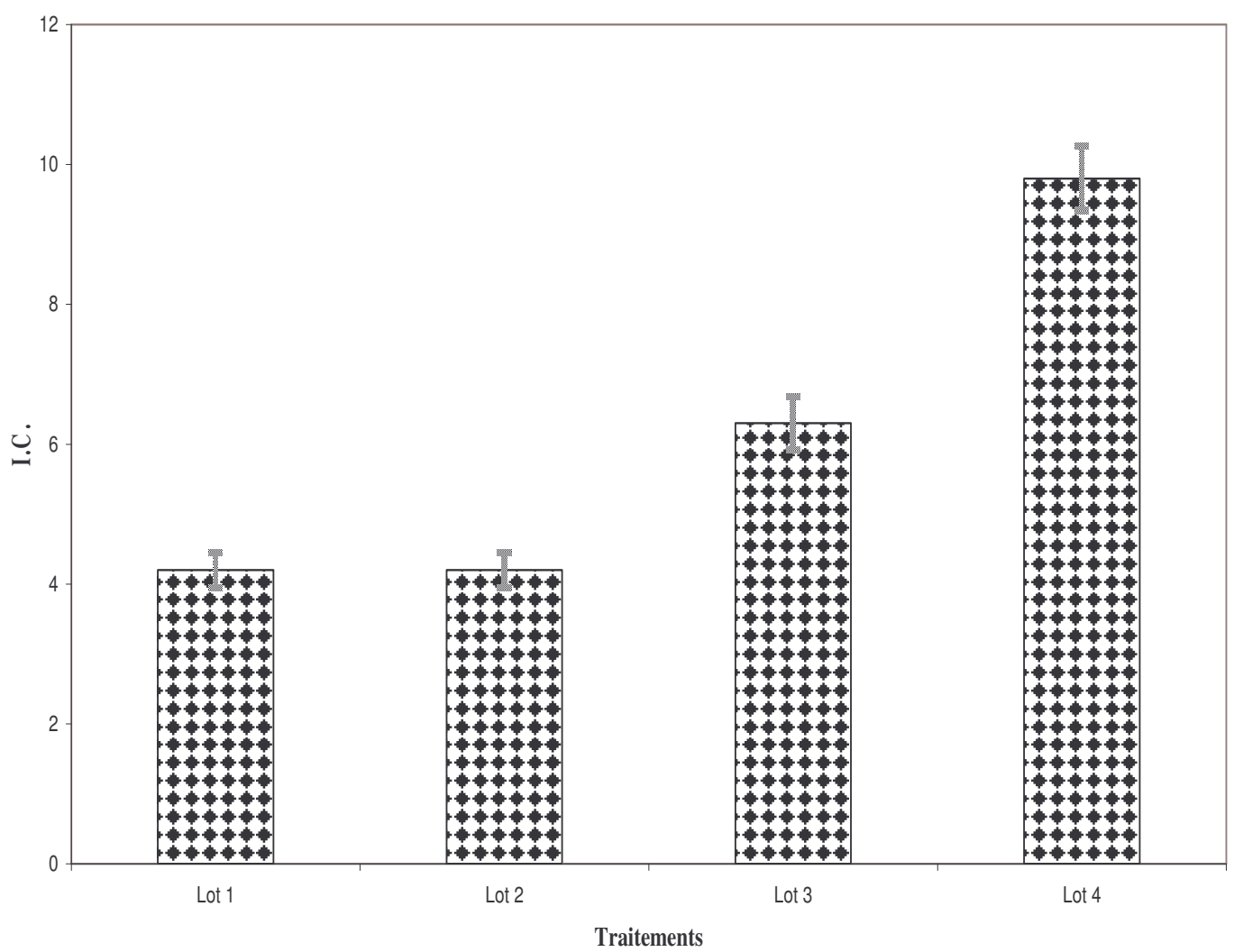

Figure 4 : Histogrammes des indices de consommation moyens des porcs par lot.

par INRA (1984) et Eusebio (1980) qui sont respectivement de 3500 et $2900 \mathrm{kcal} / \mathrm{kg}$ d'aliment. Par contre, ce taux est supérieur à celui de Kpadonou (1990) qui est de 2600 $\mathrm{kcal} / \mathrm{kg}$ d'aliment pour un régime dosant $16 \%$ de protéique. Selon Nonfon et al. (1994), les taux énergétiques recommandés par les différents auteurs semblent trop élevés pour les porcs améliorés et par surcroît pour les porcs locaux dans les pays tropicaux caractérisés par une température et un taux d'humidité élevés. Il ressort de tout ceci que le rapport (protéine / énergie) recommandé par INRA (1984) qui est de $47 \mathrm{~g} / 1000 \mathrm{kcal}$ d'énergie digestible n'est pas respecté du fait de notre restriction énergétique.

La provende de base du lot témoin (lot 1) a un taux protéique de $14,99 \%$. Ce taux protéique est inférieur à ceux recommandés par INRA (1984) et Babatundé et al. (1975) qui sont respectivement de $19 \%$, puis de 16 à $18 \%$ chez des porcs améliorés. Ce taux est par contre comparable à celui de Fetuga et al. (1974) puis de Houanye (1996) qui ont obtenu un gain de poids et une efficacité alimentaire significatifs chez les porcs locaux à un plus faible niveau protéique de la ration (respectivement $12 \%$ à $14 \%$ puis $13 \%$ ) et pour des poids initiaux similaires à ceux de nos porcs expérimentaux. En effet, Bressami (1973) et Ilori (1974) cités par Kpadonou (1990) ont rapporté que les besoins protéiques des porcs indigènes sont plus faibles que ceux des porcs importés.

Les taux protéiques élevés de nos aliments expérimentaux surtout au niveau du lot $3(19,43 \%)$ et du lot $4(21,74 \%)$ sont dû 
aux taux élevés d'incorporation d'Azolla. Les faibles performances obtenues au niveau de ces lots au cours de notre essai pourraient être également attribuées à ces taux protéiques élevés. En effet, selon Leroy (1937) cité par Aho (1986), l'excès de protéines dans la ration des porcs à l'engrais peut exercer sur la croissance des animaux, une action dépressive, qui se répercute sur les performances de croissance des porcs notamment sur leur indice de croissance.

Ce résultat vient corroborer les recommandations de l'ONG malgache "RAMILAMINA" qui suggère une incorporation de Azolla à la provende commercialisée à un taux inférieur à $30 \%$ en ce qui concerne l'alimentation des porcs.

\section{Performances zootechniques}

La consommation moyenne d'aliment par porc et par jour durant les 90 jours d'essai est de $0,33 \mathrm{~kg}$. Ce niveau de consommation représente environ la moitié de celui enregistré par Leroy (1937) qui est de $0,65 \mathrm{~kg}$ chez les porcs locaux nourris à un régime de $16 \%$ en protéine. Il représente également la moitié de celui de Houanye (1996) qui est de $0,63 \mathrm{~kg}$ chez les porcs locaux nourris à un régime de $13 \%$ en protéine. Ce bas niveau de consommation résulte d'une part de la restriction alimentaire stricte à laquelle les animaux sont soumis et d'autre part, aux taux protéiques élevés des rations suite à l'incorporation des taux élevés d'Azolla surtout dans les formules 3 et 4 . Oyeleke (1979) a rapporté que l'ingestion alimentaire des porcs décroît avec l'accroissement du niveau protéique et pour les porcs en phase d'engraissement il y a une nette réduction de la consommation à un seuil de $18 \%$ de protéines brutes. Cette observation est aussi en adéquation avec celles rapportée par Baker (1969), Blair (1972) cité par (Aho, 1986). Ce bas niveau d'ingestion pourrait être aussi expliqué par les taux d'humidité élevés des rations contenant Azolla (Azolla contient près de $95 \%$ d'humidité).

Le gain moyen quotidien (GMQ) obtenu pour tous les lots confondus au cours de notre expérimentation est de $62.35 \mathrm{~g} / \mathrm{j}$. Le GMQ le plus élevé de notre essai $(89,44 \mathrm{~g} / \mathrm{j})$ est obtenu pour le lot 2 (15\% Azolla). Ce GMQ qui de surcroît est supérieur à celui du lot témoin ( $0 \%$ Azolla), implique que la substitution à $15 \%$ Azolla serait la meilleure. Le GMQ obtenu au terme de notre essai est inférieur de $56 \%$ à celui obtenu sur les porcs locaux par Oyeleke (1979) dans la ferme de la faculté des sciences agronomiques. Ce résultat est également inférieur de 49,8\% à celui obtenu sur les porcs locaux par Houanye (1996). Par contre, il est similaire à celui de Kpadonou (1990) sur les porcs locaux et qui est en moyenne de $65 \mathrm{~g} / \mathrm{j}$ avec un régime alimentaire relativement faible en énergie (2600 kcal/kg d'aliment).

Les faibles valeurs du GMQ enregistrées au cours du présent essai, surtout avec les porcs soumis aux formules 3 et 4 (respectivement $30 \%$ et $45 \%$ Azolla), seraient dues au déséquilibre en principes alimentaires de ces formules et au déficit en énergie ingérée suite au rationnement auquel les porcs ont été soumis. En effet, selon Molenat et Legault (1986), la restriction énergétique réduit considérablement le GMQ et augmente l'indice de consommation.

Pour tous les essais, nous avons obtenu un indice de consommation moyen de 6,24. Ceci est presque le double de celui enregistré par Fetuga et al. (1974) cités par Kpadonou (1990) sur les porcs Nigérians. Cet indice de consommation (IC) est plus élevé que celui observé par Ayena (1989) et qui est de 4,2 sur les porcs locaux. Remarquons néanmoins que l'indice de consommation des lots 1 et $2(4,2)$ est le même que celui observé par Ayena (1989). Cet indice de consommation des lots 1 et 2 qui est le plus faible témoigne bien de l'efficacité de la formule 2. Les valeurs élevées de l'indice de consommation surtout 
Tableau 4: Analyse économiques des résultats.

\begin{tabular}{lllll}
\hline Paramètres & \multicolumn{1}{c}{ LOT 1 } & LOT 2 & LOT 3 & LOT 4 \\
& Témoin & & & \\
\hline & & & & \\
Coût d'un kg d'aliment sec (Fcfa) & $115,18 \mathrm{a}$ & $97,91 \mathrm{~b}$ & $80,63 \mathrm{c}$ & $63,35 \mathrm{~d}$ \\
Indice de consommation (moyenne) par porc & $4,22 \mathrm{a}$ & $4,22 \mathrm{a}$ & $6,30 \mathrm{~b}$ & $9,8 \mathrm{c}$ \\
Coût d'alimentation d'un kg de gain de poids & $486,06 \mathrm{a}$ & $412,76 \mathrm{~b}$ & $507,96 \mathrm{c}$ & $620,83 \mathrm{~d}$ \\
Gain de poids moyen total par porc (kg) & $6,97 \mathrm{a}$ & $8,05 \mathrm{~b}$ & $4,525 \mathrm{c}$ & $2,92 \mathrm{~d}$
\end{tabular}

LOT1 : témoin, LOT2 (15\% Azolla), LOT3 (30\% Azolla), LOT4 (45\% Azolla); différentes lettres sur une même ligne traduisent une différente significative au seuil de $5 \%$.

au niveau des lots 3 et 4 pourraient être essentiellement attribuées aux facteurs tels que le déséquilibre en principes alimentaires (rapport protéine / énergie non adéquat) suite à l'incorporation de taux élevés d'Azolla, au taux d'humidité élevé de Azolla incorporé, ainsi que le stade de croissance, la valorisation des aliments ingérés n'étant effective qu'à partir de $10 \mathrm{~kg}$ de poids vif chez ces porcs (Nonfon et al., 1994).

\section{Aspect économique}

En tenant compte du taux d'incorporation d'Azolla et des indices de consommation correspondants (Tableau 4), le meilleur coût d'alimentation (411,78 Fcfa ) pour un $\mathrm{kg}$ de gain de poids est enregistré au niveau du lot 2 (15\% Azolla).

\section{REMERCIEMENTS}

Cette étude a été réalisée grâce à l'appui financier de la Direction Générale de la Coopération Internationale du Royaume de Belgique dans le cadre de la Coopération Universitaire Institutionnelle CIUF-UNB.

\section{REFERENCES}

Aho JC. 1986. Substitution de la farine de poisson par la levure de bière ou le tourteau d'arachide dans l'alimentation du porc à l'engrais. Performances zootechniques, caractéristiques de carcasse et implications économiques. FSA/UNB, Abomey-calavi, 95p.

Ayena SA. 1989. Essai comparatif de quatre rations pour l'engraissement du porc local. Sékou-CPAII, 53p.

Babatundé G, Fetuga B, Oyenuga V, Ayoade A. 1975. The effect of graded levels of brewer's dried grains and maize cobs in the diets of pigs on their performance characteristics and carcass quality. Nigeria, Amin. Prod, 2: 119- 113.

Eusebio JA. 1980. Pig Production in the Tropics. Longman Group LTH: Hong Kong; 115p.

Herd YR, Cutter ED, Watanabe I. 1989. The effects of temperature and selected growth-regulating substances on sporulation in the aquatic fern Azolla. American Fern Journal, 79: 136-142.

Holnes D. 1994. Le Porc. Ed. Maisonneuve et Larose : Paris ; 221p.

Fetuga B, Babatunde G, Oyenuga V. 1974. Some Nigeria Protein concentrates as foods and feeds. Nigeria. Amin. Prod, 1 : $132-142$.

Fiogbé ED, Gangbazo KH. 2005. Production porcine avec Azolla. Actes des 2èmes Journées Scientifiques Internationales 
des Universités Nationales du Bénin. (13-16 avril 2004) ; 142-154.

Houanye KC. 1996. Etude comparative de trois rations de résidus alimentaires en fonction de la croissance du porc de race locale à Porto-Novo. CPU/PA, Abomeycalavi, 51p.

INRA 1984. Alimentation des monogastriques: Porc, Lapin, Volaille. Paris, 282p.

Kpadonou AI. 1990. Pratiques alimentaires des porcs locaux dans le district de Bopa. Performances zootechniques, caractéristiques de carcasse et implications économiques. FSA/UNB, Abomey-calavi, 178p.

Leroy A. 1937. Le Porc: les Aspects Nouveaux d'une Exploitation Lucrative. Ed. Hachette : Paris, 307p.
Molenat M, Legault C. 1986. Le porc dans les pays en voie de développement: quelques pistes d'amélioration. Paris, bulletin techn. d'info. $\mathrm{N}^{\circ} 406$, Janvier 1986, 28p.

Nonfon WR, Deka E, Adegbidji A, Codjo B. 1994. L'élevage du porc local dans le sud Bénin. Enquêtes diagnostiques sur système d'élevage et filière de commercialisation, 59p.

Oyeleke MO. 1979. A re-assessment of the dietary protein and energy levels forweaner grower ( $\mathrm{LW} \times \mathrm{L}$ ) pig in the humid lowland tropics. Ph.D Thesis, University of Ibadan, p $68-69$.

Serres H. 1989. Précis d'Elevage du Porc en Zone Tropicale. I.E.M.V.T.: Paris ; $331 \mathrm{p}$.

Van Hove C. 1989. Azolla, ses Emplois Multiples, son Intérêt en Afrique. Publication F.A.O : Belgique ; 53p. 